\title{
Prevalence of Risk Factors for Diabetes Mellitus and Hypertension Among Adult in Tabuk- Kingdom of Saudi Arabia
}

\section{Nagwa Mohammad Ahmed*}

Surgical Nursing, Department of Nursing, University College of Umulj, University of Tabuk, Kingdom of Saudi Arabia

*Corresponding Author: Dr. Nagwa Mohammad Ahmed, Surgical Nursing, Department of Nursing, University College of Umulj, University of Tabuk, Kingdom of Saudi Arabia, E-mail: n-mohamed@ut.edu.sa

Received: 11 October 2018; Accepted: 30 October 2018; Published: 12 November 2018

\begin{abstract}
Background: The worldwide incidence of diabetes has increased significantly, with the number of people of all ages with diabetes expected to approximately double between 2000 and 2030, and is seen as a major source of disability and health costs. The balance between prevention and intervention, as with other infectious diseases, is the best way to stop the growing burden of cardiovascular disease.
\end{abstract}

Objective: This study evaluates the prevalence of risk factors for diabetes and hypertension in a sample from Tabuk University.

Methods: A cross-sectional study was conducted in 2018 with 120 male and female students at Tabuk University (Tabuk, Saudi Arabia) between 20-55 years of age from various departments at Tabuk University.

Results: The evaluation was conducted with 120 participants to assess of prevalence for hypertension and diabetes at Tabuk University, where the prevalence rate is already higher than most cities in Saudi Arabia. Ten per cent of participants had high blood sugar and 10 per cent developed problems in this area, with no significant difference between males and females. 
Conclusion: This study display that cardiovascular risk factors will affect those with major main health disorders in the future. Thus, a national campaign to promote healthy eating, better lifestyles, and physical activity is the priority of health care.

Keywords: Cardiovascular Risk Factors; Hypertension; Diabetes Mellitus; Saudi Arabia

\section{Introduction}

Cardiovascular diseases (CVD) are one of the badly common chronic diseases that cause death and are a primary source of chronic disability and health costs [1]. Key risk factors for CVD include hypertension, high blood sugar, serum lipid abnormalities, smoking, obesity, low fruit/vegetable intake, and physical inactivity. Studies mention that these risk factors are popular worldwide. Most developing countries in the Middle East are undergoing an epidemiological transition, from infectious diseases to chronic diseases [2]. These countries are also undergoing a major shift in lifestyles patterns in relation to dietary habits, physical activity and some customs (such as smoking). In Saudi Arabia, there is little information on the impact of changes in lifestyle such as reducing physical activity and dietary habits on the prevalence of diabetes, hypertension and hyperlipidemia. Thus, these changes are occurring rapidly in Saudi Arabia. Cardiovascular disease can be prevented if the community receives information, education and appropriate communication about potential risk factors. Most risks are attributed to lifestyle patterns and behavioral patterns and can be changed $[3,4]$. Therefore, deciding the charge of risk factors for cardiovascular disease in the population helps to design and implement promotional and preventive measures. In the developing world, there is a wide gap between chronic disease burden and response. If the emergence and prevention of unguided risk factors is left, the growth of the problem will continue to accelerate [5, 6]. Therefore, the use of prevention and control interventions as in other infectious diseases will be the best way forward $[4,5]$.

\section{Objectives}

This study aimed to set the prevalence of diabetes and hypertension among adults aged 20 years and over in a sample taken from students and staff at Tabuk University. The secondary objective was to border the relationship between the prevalence of these factors and some demographic, environmental, social, cultural and economic factors.

\section{Literature Review Hypertension}

In the review article Prevalence for hypertension and obesity in patients with type 2 diabetes mellitus in observational studies: a systematic revision of hypertension literature in an article reviewing the prevalence for hypertension and obesity in patients with type 2 diabetes in observational studies: A review of methodological literature achieved by Colosia et al. [7] was discussed the relationship between diabetes and hypertension. It was found that diabetic patients are very likely to have hypertension as well. Diabetes and hypertension have been linked to each other, meaning that the root cause is obesity. Through the literary research of various databases has been 
expolred the relationship between hypertension and obesity with diabetes type II. 8. Saeed et al. [8] include various variables related to the prevalence, control, sensbility and treatment of hypertension.

Saeed et al. [8] have different variables related to the prevalence, control, sensbility and treatment of high blood pressure. Article Saeed et al. [8] includes various variables related to the prevalence, control, sensbility and treatment of high blood pressure. During the study with a population sample, predictive factors were determined based on sex, environment, social environment, physical activity and obesity. Although high blood pressure is found to be high among the population, sensbility and control practices are very low, requiring an sensbility program to combat proliferation in the population of Saudi Arabia. Another important study, Pre-Hypertension among young females in Dammam, was carried out by Koura et al. [9]. This study examines the prevalence of high blood pressure in females by studying the population sample from four universities in Dammam. By conducting the questionnaire $13.5 \%$ of females, they had a condition of high blood pressure, they were unaware of it. Sources of this prevalence are physical inactivity, obesity, and unhealthy lifestyle. Diabetes Study of Diabetes in Saudi Arabia by Nazah et al. [10], by conducting the questionnaire $13.5 \%$ of females, they had a condition of high blood pressure, they were unaware of it. Sources of this prevalence are physical inactivity, obesity, and unhealthy lifestyle. Diabetes Study of Diabetes in Saudi Arabia by Nazah et al. [11], by conducting the questionnaire $13.5 \%$ of females, they had a condition of high blood pressure, they were unaware of it. Sources of this prevalence are physical inactivity, obesity, and unhealthy lifestyle. Diabetes Study of Diabetes in Saudi Arabia by Nazah et al. [11].

\section{Diabetes Mellitus}

Diabetes study in Saudi Arabia conducted by Al-Nazha et al. [11], the prevalence of diabetes in the Saudi population was determined by both sexes as well as rural and urban communities. It covers the causes and environmental and physiological factors that have led to the disease. In addition, the study also identifies various health risks associated with diabetes mainly in coronary artery disease (CAD). Another study on the prevalence of diabetes in rural Saudi Arabia by Fatani in 2004, the prevalence of diabetes in the Saudi population was determined by both sexes as well as rural and urban communities. It covers the causes and environmental and physiological factors that have led to the disease. In addition, the study also identifies various health risks associated with diabetes mainly in coronary artery disease (CAD). Another study on the prevalence of diabetes in rural Saudi Arabia by Fatani, focuses on the incidence of diabetes in rural areas of Saudi Arabia.

This study addresses the various causes of diabetes and its underlying causes in different environmental settings such as rural and urban areas. Through the statistical analysis of BMI and blood glucose level in relation to the economic and social status of the different sample of the sample, the effect of environmental factors was studied. To understand the prevalence of diabetes in Saudi Arabia, a study entitled 'The prevalence of diabetes in Saudi males' by Bacchus et al. [12] was conducted to identify any genetic phenomena and inference of the disease. The relationship between weight, age, tobacco consumption and environmental status at the onset of diabetes in these males. 


\section{Methods}

\subsection{Design and setting}

A cross-sectional study was conducted with 120 employees and students at Tabuk University, Umuljj city funded by the Kingdom of Saudi Arabia during 2018. The age of 20 years or more (60 males and 60 females) from different faculties at Tabuk University. The cross-section design facilitates the monitoring of some subsets of the population at the same time with respect to independent variables (Bollett and Beck, 2004). Correlation study is an effective and effective design for collecting large amounts of data in relation to certain phenomena [13].

\subsection{Population and sample}

Population and Sample the sample size was subjected to a power analysis using a power estimation of 0.80 and a medium effect size for a two-tailed test with alpha=0.05 as the minimum. The adequate sampling size for the study was 120 respondents. As a result, a random sample of male and female university staff was recruited for interviews using a questionnaire, a clinical examination and a body/laboratory measurements. The largest division was by gender. These groups were sub- categorized into four age groups.

\subsection{Instruments and techniques}

There were equal numbers in the sex categories, but males and females were not equal in the total population; their relative proportions differed with age. Tools and Techniques We developed a self-administered questionnaire with 25 questions and used a 4-point Likert scale to assess participants in age, gender, level of education, socioeconomic status and risk factors for cardiovascular disease (family history and pre-existing medical conditions such as diabetes and hypertension). The result was cronbach Alpha at 0.825 .

Blood pressure measurements were performed in the morning when the participant arrived at the study sites. Participants were advised not to eat or drink anything before the measurements, and make sure participants did not consume coffee or smoke before reaching school because this may affect blood pressure measurements. Blood pressure was measured using a mercurial blood pressure meter (KBM, sm-500, Japan). Two blood pressure reading were taken on the upper left arm with the participant in the sitting position after at least 5 to 10 minutes of rest. The average reading was used in this analysis. Hypertension is defined as=140/90 $\mathrm{mmHg}$ according to the WHO classification of hypertension [14].

\subsection{Data collection}

Different methods of data analysis were performed including descriptive analysis and inferential statistical analysis. Descriptive statistics for the demographic data and other questionnaire items were presented using means, standard deviations and P-values. A variety of statistical tests were used to identify the differences between groups such as the independent sample t-test and analysis of one-way variance (ANOVA). The sample size was calculated to identify the main outcome variables being measured as well as the instrument used to measure those variables and the anticipated differences between groups [15]. An average size effect was expected because this is a very common 
practice in the field of study [16]. It has been argued that obtaining more responses from the survey site increases its statistical power [17].

\section{Ethical Approval}

Ethical approval for the study was obtained from the research ethical committee of the University of Tabuk on Sep. 20, 2018, Number: S-1439- 0028. Each participant was provided with an information sheet that clarified the aim of the study, the rights of the participants, the assessment process, the process for completing the survey and the use of the collected data. The information sheet also stated that all information provided by the participants would remain unknown and that privacy would be guaranteed. The information sheet also provided contact details for any queries. The participants were advised about the voluntary nature of participation in the study and informed that they had the option to refuse without penalty or loss of benefit(s). Also, it was made that the study findings would be presented in a format that makes it impossible to identify the participants.

\section{Results}

This study aimed the prevalence of risk factors for hypertension and diabetes among staff aged 20 at crucial years and over in a sample taken from employees, students and employees of Tabuk University. 120 employees and students at Tabuk University were studied, 60 (50\%) females and 60 (50\%) males with four age groups, $21.7 \%$ of the subjects. Most of the participants in the third group (46-55 years) and the fourth group were the first group 20-35 and accounted for $20.8 \%$ of the persons. The second group was 36-45 years old and the age group (55 years) was $33.3 \%$ and $24.2 \%$, respectively. In addition, $62.5 \%$ of respondents were Saudis and 37.2\% were non-Saudis (Table $1)$.

\begin{tabular}{|l|l|l|}
\hline Demographics & Frequency & Percent \\
\hline Gender & 60 & $50 \%$ \\
\hline Male & 60 & $50 \%$ \\
\hline Female & \multicolumn{2}{l|}{} \\
\hline Age & 25 & $20.8 \%$ \\
\hline$(20-35)$ & 26 & $21.7 \%$ \\
\hline$(36-45)$ & 40 & $33.3 \%$ \\
\hline$(46-55)$ & 29 & $24.2 \%$ \\
\hline above 55 & \multicolumn{2}{|l|}{} \\
\hline Level of education & 24 & $20 \%$ \\
\hline School & 22 & $18.3 \%$ \\
\hline Diploma & 13 & $10.8 \%$ \\
\hline Undergraduate & 61 & $50.8 \%$ \\
\hline Graduate & \multicolumn{2}{|l|}{} \\
\hline Job Position & \multicolumn{2}{|l|}{} \\
\hline
\end{tabular}




\begin{tabular}{|l|l|l|}
\hline Employees & 58 & $48.3 \%$ \\
\hline Students & 62 & $52.7 \%$ \\
\hline Nationality & \multicolumn{2}{|l|}{} \\
\hline Saudi & 75 & $62.5 \%$ \\
\hline Non Saudi & 45 & $37.5 \%$ \\
\hline Marital Status & \multicolumn{2}{|l|}{} \\
\hline Single & 28 & $23.3 \%$ \\
\hline Married & 80 & $66.7 \%$ \\
\hline Divorce & 7 & $5.8 \%$ \\
\hline Widowed & 5 & $4.2 \%$ \\
\hline
\end{tabular}

Table 1: Participant Demographics.

There were fixed additions with both ages. Mean systolic and diastolic blood pressure was no significant difference in the mean for males and females as a function of age. The fasting sugar test showed that $10 \%$ of the participants had hyperglycemia, and $10 \%$ were on the borderline with no statistically significant differences between males and females. Thus, this study shows an increase in the prevalence of diabetes compared to previous Saudi studies. Fasting sugar testing showed that $10 \%$ of the participants were hyperglycemic, and $10 \%$ were on the borderline with no significant differences between males and females (Table 2). Thus, this research shows an increase in the prevalence of diabetes mellitus versus previous Saudi studies.

\begin{tabular}{|l|l|l|}
\hline Laboratory Blood Tests & Frequency & Percent \\
\hline Blood Pressure & 95 & $79.2 \%$ \\
\hline Less than 140/90 & 25 & $10.8 \%$ \\
\hline More than 140/90 & \multicolumn{2}{l|}{} \\
\hline Fasting Blood Sugar & 96 & $72.5 \%$ \\
\hline $70-99 \mathrm{mg} / \mathrm{dl}$ & 12 & $10 \%$ \\
\hline $100-126 \mathrm{mg} / \mathrm{dl}$ & 12 & $10 \%$ \\
\hline More than $126 \mathrm{mg} / \mathrm{dl}$ & &
\end{tabular}

Table 2: Frequency and Percentage of Laboratory Blood Tests.

Table 3 present statistical analysis with ANOVA values for results of the relationship between age with blood pressure, fasting blood sugar, Table 4 shows that blood pressure ANOVA analysis among different age groups had $\mathrm{F}$ and P-values of 3.62 and 0.015 , respectively. This is a significant relationship and demonstrates that there is an impact of age on blood pressure. Table 4 also showed that ANOVA analysis between different age groups with 
fasting blood sugar. Here, F and P-values were 2.30 and 0.081 respectively, which demonstrate the non- significant relationship between different age groups and fasting blood sugar.

\begin{tabular}{|l|l|l|}
\hline & F & P-value \\
\hline Blood Pressure & 3.62 & 0.015 \\
\hline Fasting Blood Sugar & 2.30 & 0.081 \\
\hline
\end{tabular}

Table 3: ANOVA Analysis with Age Groups.

Table 5 displays statistical analysis with Independent Sample T Test values for findings for the relationship between job with blood pressure, fasting blood sugar. The Independent Sample $\mathrm{T}$ Test analysis between students and employee groups for blood pressure had $\mathrm{T}$ and P-values of 2.72 and 0.046 , respectively (Table 4). This shows a significant relationship and demonstrates the impact of job on blood pressure. Detailed Independent Sample T-Test statistics of the research participants including the relationship between job and diabetes.

\begin{tabular}{|l|l|l|}
\hline & T & P-value \\
\hline Blood Pressure & 2.72 & 0.046 \\
\hline Fasting Blood Sugar & 2.93 & 0.035 \\
\hline
\end{tabular}

Table 4: T-Test of participants regarding their job related to hypertension and diabetes.

\section{Discussion}

This study measured the prevalence of CVD risk factors. The study was validated with the WHO STEPS rules and testing strategies that included a straightforward and irregular examining method. This study is the first of its kind in the nation to attempt all three segments of WHO STEPS in a group setting. The low response rate might influence the genuine dispersion of the risk factors, and the results should be translated with caution. The prevalence of hyperlipidemia is growing significantly and is one of the main contributors to many diseases due to its physiological association with other cardiovascular risk factors such as diabetes and hypertension [18]. The dominance of hypertension (10.8\%) is similar to that in Butajira, Ethiopia [20] and meta-analysis (25 studies) from 10 sub-Saharan Africa [19] our value is lower than in.

In Addis Ababa, Ethiopia [20] and the systematic review of studies in Europe and North America [21]. The conceivable objectives behind this distinction may be different types of study in social, demographic and economic characteristics. The prevalence of hypertension was more than twice that of urban areas than rural areas. This is consistent with the findings in Ethiopia [20] and the systematic review of sub-Saharan studies [19]. No significant differences were observed between males and females in terms of hypertension, these contrasts with the Ethiopian study and the review of European and North American research [21]. 
However, the findings in the Sub-Saharan Africa Review do not refer to credible cross-country discrimination [19]. This study mention that hypertension affects less than a quarter of participants, which agrees with an earlier national study [10]. Of note, our mode age is 10-15 years young than that in corresponding studies in developed nations [22, 23]. This demonstrates the worldwide burden of hypertension is extensive and expanding suggesting the need for urgent intervention. This study showed significant relationship to hypertension with age in both sexes and with national and global studies in many populations the different geographic, social, and financial attributes [24, 25].

Globally, diabetes prevalence will increase by $42 \%$ from 2003 to 2025 [26]. The prevalence rate in the Gulf region is the highest in Bahrain (25.7\%) and Oman (16.1\%). This study indicated further escalation in the prevalence of diabetes in collaboration with previous studies conducted in Saudi Arabia. The dramatic rise in the diabetes mellitus prevalence can be clarified by the disorder of advanced years. About $29 \%$ of the participants over 55 have diabetes. Studies carried out in Saudi Arabia have diverse age-specific prevalence rates. This study shows an increase in diabetes mellitus prevalence in the elderly. Indeed, this relationship between diabetes and age is consistent with earlier studies $[11,27]$. The prevalence of diabetes mellitus (10\%) and borderline diabetes $(10 \%)$ is similar to the results of Al-Nozha et al. [11] who recognized the prevalence of diabetes mellitus in the Saudi population in both males and females. Another study by Fatani et al. [28] reported the rate of diabetes mellitus in rural Saudi Arabia both studies were consistent with this study. Worldwide, the prevalence of diabetes is similar in males and females, but slightly higher in females at $<60$ years of age and in males $<60$ years. This is not detected in this search.

\section{Conclusion}

In summary, the prevalence of hypertension and diabetes in the city of Umuljj, Tabuk University, is higher than in most cities in Saudi Arabia. The prevalence of cardiovascular risk factors is higher than in other previous Saudi studies. Despite the presence of a younger population, the city of Tabuk Umuljj has a high prevalence of cardiovascular risk factors. In fact, even higher than the European countries and North America. This means that there will eventually be an increase in the prevalence of hypertension and diabetes with the population unless preventive policies are introduced. Data indicate that cardiovascular risk factors affect individuals and will be significant health disorders in the future. Thus, a nationwide campaign should be promoted to promote a healthy diet and a healthy lifestyle.

\section{Acknowledgment}

The authors would like to acknowledge the financial support of this work, from the Deanship of Scientific Research (DSR), Tabuk University, under Grant No. S-1439-0028. Also, we would like to thank the participants at Tabuk University in Umulij, Saudi Arabia, for this study was not possible without their assistance.

\section{References}

1. Strong K, Mathers C, Leeder S, et al. Preventing chronic diseases: how many lives can we save?. The Lancet 366 (2005): 1578-1582. 
2. Santosa A, Wall S, Fottrell E, et al. The development and experience of epidemiological transition theory over four decades: a systematic review. Global health action 7 (2014).

3. World Health Organization. Regional Office for Europe. The European health report 2005: Public health action for healthier children and populations. WHO Regional Office Europe (2005): 97.

4. World Health Organization. Preventing chronic diseases: a vital investment. Geneva: WHO (2012).

5. Jamison DT, Breman JG, Measham AR, et al. Disease control priorities in developing countries. World Bank Publications (2006).

6. Unwin N, Setel P, Rashid S, et al. Noncommunicable diseases in sub-Saharan Africa: where do they feature in the health research agenda?. Bulletin of the World Health organization 79 (2001): 947-953.

7. Colosia AD, Palencia R, Khan S. Prevalence of hypertension and obesity in patients with type 2 diabetes mellitus in observational studies: a systematic literature review. Diabetes, metabolic syndrome and obesity: targets and therapy 6 (2013): 327.

8. Saeed AA, Al-Hamdan NA, Bahnassy AA, et al. Prevalence, sensbility, treatment, and control of hypertension among Saudi adult population: a national survey. International journal of hypertension (2011).

9. Koura MR, Al Dabal BK, Rasheed PA, et al. Prehypertension among young adult females in Dammam, Saudi Arabia (2012).

10. Al-Nozha MM, Abdullah M, Arafah MR, et al. Hypertension in Saudi Arabia. Saudi medical journal 28 (2007): 77-84.

11. Al-Nozha MM, Al-Maatouq MA, Al-Mazrou YY, et al. Diabetes mellitus in Saudi Arabia (2004).

12. Bacchus RA, Bell JL, Madkour M, et al. The prevalence of diabetes mellitus in male Saudi Arabs. Diabetologia 23 (1982): 330-332.

13. Polit DF, Beck CT. Nursing Research: Generating and Assessing Evidence for Nursing Practice (9th Edn). Philadelphia: Lippincott Williams and Wilkins (2012).

14. NIH. The seventh report of the joint national committee on prevention, detection, evaluation and treatment of hypertension (JNC 7). National Institutes of Health (2008).

15. Gerrish K, Lacey A. The Research Process in Nursing. (6th Edn) Oxford: Wiley-Blackwell (2010).

16. Watson R, McKenna H, Cowman S, et al. Nursing Research: Designs and Methods, Edinburgh, Churchill Livingstone Elsevier (2008).

17. Kemper E, Stringfield S, Teddlie C. Mixed methods sampling strategies in social science research. In Tashakkori A, Teddlie C, Handbook of mixed methods in social and behavioral research (2003): 273-296.

18. Mehio Sibai A, Nasreddine L, Mokdad AH, et al. Nutrition transition and cardiovascular disease risk factors in Middle East and North Africa countries: reviewing the evidence. Annals of Nutrition and Metabolism 57 (2010): 193-203.

19. Addo J, Smeeth L, Leon DA. Hypertension in Sub-Saharan Africa a systematic review. Hypertension 50 (2007): 1012-1018.

20. Tesfaye F. Epidemiology of cardiovascular disease risk factors in Ethiopia: the rural-ruban gradient (2008). 
21. Wolf-Maier K, Cooper RS, Banegas JR, et al. Hypertension prevalence and blood pressure levels in 6 European countries, Canada, and the United States. Jama 289 (2003): 2363-2369.

22. Davis BR, Cutler JA, Gordon DJ. Major outcomes in high risk hypertensive patients randomized to angiotensin-converting enzyme inhibitor or calcium channel blocker vs diuretic: The Antihypertensive and Lipid Lowering treatment to prevent Heart Attack Trial (ALLHAT). Jama 288 (2002): 2981-2997.

23. Perkovic V, Huxley R, Wu Y, et al. The burden of blood pressure-related disease a neglected priority for global health. Hypertension 50 (2007): 991-997.

24. Erem C, Hacihasanoglu A, Kocak M, et al. Prevalence of prehypertension and hypertension and associated risk factors among Turkish adults: Trabzon Hypertension Study. Journal of public health 31 (2009): 47-58.

25. SIngh RB, Fedacko J, Pella D, et al. Prevalence and risk factors for prehypertension and hypertension in five Indian cities. Acta cardiológica 66 (2011): 29.

26. Gan D. International Diabetes Federation: Diabetes Atlas (2003).

27. Wild SH, Roglic G, Green A, et al. Global prevalence of diabetes: estimates for the year 2000 and projections for 2030 response to Rathman and Giani. Diabetes care 27 (2004): 2569-2569.

28. Fatani HH, Mira SA, El-Zubier AG. Prevalence of diabetes mellitus in rural Saudi Arabia. Diabetes care 10 (1987): 180-183.

Citation: Nagwa Mohammad Ahmed. Prevalence of Risk Factors for Diabetes Mellitus and Hypertension Among Adult in Tabuk- Kingdom of Saudi Arabia. Archives of Clinical and Medical Case Reports 2 (2018): 206-215.

(CC) This article is an open access article distributed under the terms and conditions of the
Creative Commons Attribution (CC-BY) license 4.0 